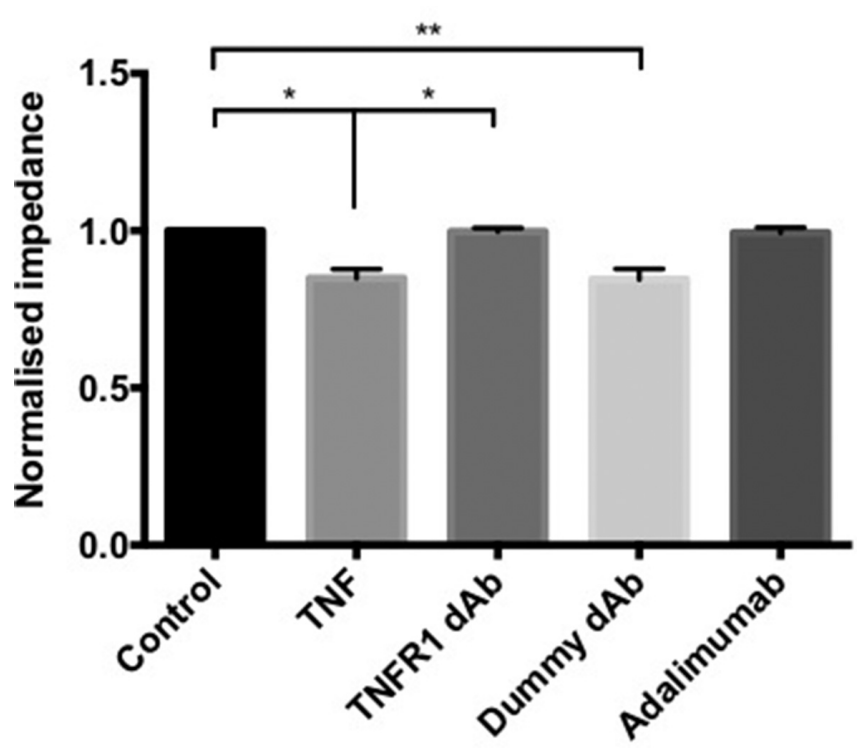

Abstract P19 Figure 1 A549 cells plated on an iCelligence 8-well gold electrode coated plate were incubated with TNFR1 dAbTM, a dummy $\mathrm{dAb}$ or Adalimumab ${ }^{\mathrm{TM}}$ for $1 \mathrm{~h}$ then exposed to exogenous TNF or vehicle control. Electrical impedance was measured continuously over $50 \mathrm{~h}$. Trough normalised impedance was measured over $50 \mathrm{~h}$ post treatment $(n=3-5)$. Data are presented as mean \pm SEM analysed by Kruskal-Wallis (Dunns). ${ }^{*} p<0.05,{ }^{* *} p<0.01$

be due to disruption of epithelial junctional proteins; we speculate that this may alternatively be due to TNFR1 induced cell death.

\section{P20 DELINEATING THE CONTRIBUTION OF FORMYLATED PEPTIDES AND FORMYL PEPTIDE RECEPTOR 1 TO THE PATHOGENESIS OF ACUTE LUNG INJURY}

${ }^{1} \mathrm{DA}$ Dorward, ${ }^{1} \mathrm{CD}$ Lucas, ${ }^{2} \mathrm{MK}$ Doherty, ${ }^{1} \mathrm{~GB}$ Chapman, ${ }^{1} \mathrm{E}$ Scholefield, ${ }^{1} \mathrm{~A}$ Conway-Morris, ${ }^{1} \mathrm{~T}$ Kipari, ${ }^{1} \mathrm{CT}$ Robb, ${ }^{1} \mathrm{JM}$ Felton, ${ }^{2} \mathrm{PD}$ Whitfield, ${ }^{1} \mathrm{C}$ Haslett, ${ }^{1} \mathrm{~K}$ Dhaliwal, ${ }^{1} \mathrm{AG}$ Rossi. ${ }^{1} \mathrm{MRC}$ Centre for Inflammation Research, University of Edinburgh, Edinburgh, UK; ${ }^{2}$ University of Highlands and Islands, Inverness, UK

\subsection{6/thoraxjn-2014-206260.170}

Background Acute respiratory distress syndrome (ARDS) remains an often fatal condition without effective pharmacological therapies. Characteristically, a neutrophil-dominant disorder, it is associated with a dysregulated inflammatory response and tissue injury. Neutrophil migration into inflammatory sites is controlled by a variety of factors; in sterile tissue injury mitochondrial formylated peptides are released following necrotic cell death and bind to formyl peptide receptor 1 (FPR1) on neutrophils to induce migration and activation.

Hypothesis That mitochondrial formylated peptides are elevated in ARDS and drive FPR1-mediated neutrophil recruitment. Inhibition of FPR1 in sterile lung injury would therefore attenuate the inflammatory response through multiple FPR1-mediated effects.

Methods Mitochondrial DNA and formylated peptides were quantified in plasma of ARDS patients and healthy controls by qPCR, western blot and LC-MS/MS. Healthy volunteer neutrophils were stimulated with mitochondrial formylated peptides and chemotaxis assays and flow cytometry used to assess neutrophil function. Intracellular signalling was assessed by western blotting. Mouse models of infective (E. coli) and sterile (hydrochloric acid) acute lung injury were used.

Results Free mitochondrial DNA and formylated peptides were elevated in ARDS patients. Mitochondrial formylated peptides induced FPR1-dependent neutrophil chemotaxis through PI3Kand MAPK-mediated control of the $\beta_{2}$-integrin heterodimer Mac1. In sterile acid-induced injury FPR1 inhibition resulted in reduced neutrophil migration, pulmonary haemorrhage, protein leak and pro-inflammatory cytokine expression. Furthermore, acid-induced reduction in alveolar macrophage number was inhibited while interstitial macrophages displayed an alternatively activated phenotype. FPR1 was also found to be expressed on mouse type 1 alveolar epithelial cells suggesting further possible mechanisms through which FPR1-mediated alveolar leak occurs. Importantly, delivery of FPR1 antagonists $12 \mathrm{~h}$ after injury also reduced acute lung inflammation demonstrating potential therapeutic relevance. In non-sterile E. coli-mediated lung injury partial antagonism of FPR1 resulted in reduced alveolar neutrophil numbers and attenuated vascular leak without altering bacterial clearance.

Conclusions Mitochondrial formylated peptides and FPR1 play an important role in the pathogenesis of sterile acute lung injury. This appears to be predominantly through neutrophil-dependent means but their role in macrophage and epithelial cell function could also be important. FPR1 antagonism may therefore represent a multi-cellular therapeutic target in the treatment of ARDS.

\section{P21 HYPOXIA-INDUCED NEUTROPHIL SURVIVAL IS DEPENDENT ON PHOSPHOINOSITIDE 3-KINASE (PI3-K)- MEDIATED SIGNALLING}

${ }^{1} S$ Palazzo, ${ }^{1} \mathrm{~L}$ Porter, ${ }^{1} \mathrm{JK}$ Juss, ${ }^{2} \mathrm{E}$ Hessel, ${ }^{2} \mathrm{~A}$ Amour, ${ }^{2} \mathrm{D}$ House, ${ }^{2} \mathrm{M}$ Begg, ${ }^{1} \mathrm{ER}$ Chilvers. ${ }^{1}$ University of CambridgeSchool of Clinical Medicine, Cambridge, UK; ${ }^{2}$ GlaxoSmithKline, Stevenage, UK

\subsection{6/thoraxjnl-2014-206260.171}

Introduction and objectives Neutrophils (PMNs) are a key component of the innate immune response to invading pathogens. They accumulate at sites of inflammation and infection, which are typically characterised by low oxygen tensions (e.g. in the acute respiratory distress syndrome (ARDS)). Human PMNs undergo constitutive apoptosis, their survival contingent upon pro-survival and pro-apoptotic signals derived from their microenvironment. Hypoxia profoundly delays PMN apoptosis, resulting in persistence of PMNs at inflammatory foci and this may perpetuate hypoxia-mediated lung injury. Given the importance of phosphoinositide 3-kinase (PI3-K) signalling in cytokine-mediated neutrophil survival, we hypothesised that hypoxia-induced PMN survival may also involve PI3-K-mediated signalling.

Methods Highly pure PMNs isolated from healthy volunteers were incubated for $20 \mathrm{~h}$ under normoxic $(20 \mathrm{kPa})$ and physiologically relevant hypoxic $(3 \mathrm{kPa})$ conditions with a pan-PI3-K inhibitor (LY294002 at $10 \mu \mathrm{M}$ ), a novel pan-Class I PI3-K inhibitor (ZSTK474 at $1 \mu \mathrm{M}, 3 \mu \mathrm{M}$ and $10 \mu \mathrm{M}$ ) or novel PI3-K Class I isoform-selective inhibitors (PI3-K $\delta$ at $1 \mu \mathrm{M}$; PI3-K $\gamma$ at $3 \mu \mathrm{M}$

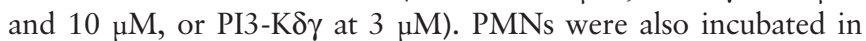
normoxia and hypoxia in the presence of GM-CSF $(1 \mathrm{ng} / \mathrm{ml})$ with the same panel of inhibitors, allowing comparison with GM-CSF mediated survival, which is largely PI3-K dependent. PMN apoptosis was assessed using two complementary techniques - morphology and flow cytometry following annexin V-FITC and propidium iodide staining. 
Results Compared with normoxia, hypoxia promoted PMN survival (mean $\% \pm$ SEM apoptotic cells at $20 \mathrm{~h} ; 30.9 \pm 1.9$ vs. $59.0 \pm 1.8$ respectively, $\mathrm{p}<0.0001$ ). Both pan-PI3-K inhibitors reversed the pro-survival effect of hypoxia in a concentrationdependent manner, LY294002 $(10 \mu \mathrm{M} ; 60.3 \pm 4.0, \mathrm{p}<0.0001)$ and ZSTK474 $(10 \mu \mathrm{M} ; 58.3 \pm 2.6, \mathrm{p}<0.0001)$ without affecting the basal rate of apoptosis. This effect was not seen with the

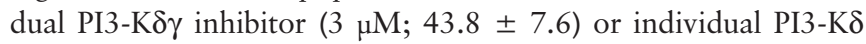
$(1 \mu \mathrm{M} ; 43.0 \pm 5.8)$ and $\gamma$ inhibitors $(3 \mu \mathrm{M} ; 34.9 \pm 4.5$ and $10 \mu \mathrm{M} ; 32.6 \pm 3.6$ ).

Conclusions Our results indicate that hypoxia-induced PMN survival is PI3-K dependent. Targeting this pathway may accelerate PMN apoptosis, resulting in resolution of inflammation.

\section{P22 ENDOPLASMIC RETICULUM STRESS MARKERS CORRELATE WITH FIBROSIS IN IDIOPATHIC PULMONARY FIBROSIS AND NON-SPECIFIC INTERSTITIAL PNEUMONIA}

${ }^{1} \mathrm{H}$ Parfrey, ${ }^{1} \mathrm{~B}$ Beardsley, ${ }^{1} \mathrm{~J}$ Knight, ${ }^{2} \mathrm{~S} J$ Marciniak, ${ }^{1} \mathrm{D}$ Rassl. ${ }^{1}$ Papworth Hospital NHSFT, Cambridge, UK; ${ }^{2}$ CIMR, University of Cambridge, Cambridge, UK

\subsection{6/thoraxjnl-2014-206260.172}

Idiopathic pulmonary fibrosis (IPF) and non-specific interstitial pneumonia (NSIP) are forms of idiopathic interstitial pneumonias that have distinct histopathological features and outcomes. It is unknown if these idiopathic interstitial pneumonias have common mechanisms of fibrosis. Endoplasmic reticulum (ER) stress has been implicated in the pathogenesis of sporadic and familial idiopathic pulmonary fibrosis. In response to endoplasmic reticulum (ER) stress, cells trigger the unfolded protein response (UPR) with upregulation of chaperones, such as BiP, and the phosphatase growth arrest and DNA damage 34 (GADD34). However, this may have profound effects on cell proliferation and survival. We hypothesised that ER stress may also be involved in the pathogenesis of NSIP.

Paraffin embedded lung biopsy sections from 4 patients with sporadic idiopathic pulmonary fibrosis (IPF) (UIP histopathology) and 4 non-specific interstitial pneumonia (NSIP) (NSIP histopathology) were evaluated for the ER stress markers BiP and GADD34 using immunohistochemistry. For each biopsy sample, six high power fields (x 200 magnification) were scored for fibrosis and inflammation as well as BiP and GADD34 using semiquantitative analysis by 2 blinded, independent investigators.

$\mathrm{BiP}$ and GADD34 were expressed in areas of fibrosis, localised to reactive type II pneumocytes and endothelial cells. No staining was detected in fibroblasts or fibroblastic foci. In sporadic IPF (UIP), levels of BiP within the epithelium correlated with fibrosis $\left(\mathrm{r}^{2} 0.56, \mathrm{p}=0.0001\right.$, Figure 1a) more than inflammation $\left(\mathrm{r}^{2} 0.38, \mathrm{p}=0.0013\right)$. In contrast, epithelial GADD34 was
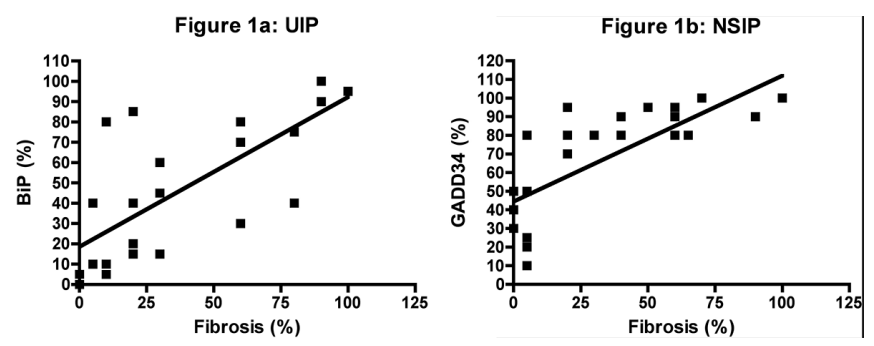

Abstract P22 Figure 1 (A) UIP, (B) NSIP more strongly associated with NSIP fibrosis $\left(\mathrm{r}^{2} \quad 0.56\right.$, $p<0.0001$, Figure 1b). There was no association between the ER stress markers and inflammation in NSIP.

These data suggest that ER stress and the unfolded protein response are features of NSIP as well as IPF and may play a role in determining the severity of the fibrotic response.

\section{P23 TARGET AND BIOMARKER DISCOVERY FOR HEDGEHOG PATHWAY ACTIVITY IN IDIOPATHIC PULMONARY FIBROSIS IN SUPPORT OF A PHASE 2 RANDOMISED, DOUBLE-BLIND, PLACEBO-CONTROLLED STUDY TO ASSESS EFFICACY AND SAFETY OF VISMODEGIB IN IPF (ISLAND)}

${ }^{1} \mathrm{G}$ Jia, 'S Chandriani, 'AR Abbas, 'D DePianto, ${ }^{1} E$ N'Diaye, ${ }^{1} \mathrm{M}$ Yaylaoglu, ${ }^{2} \mathrm{HR}$ Collard, ${ }^{2} \mathrm{P}$ Wolters, 'J Egen, ${ }^{3} \mathrm{~A}$ Scalori, ${ }^{3} \mathrm{~A}$ Ackrill, 'J Hou, ${ }^{3} \mathrm{~J}$ Kaminski, ${ }^{4} \mathrm{TM}$ Maher, ${ }^{1} \mathrm{~J} R$ Arron. ${ }^{1}$ Genentech, Inc., South San Francisco, USA; ${ }^{2}$ Division of Pulmonary and Critical Care Medicine, Department of Medicine, University of California, San Francisco, USA; ${ }^{3}$ Roche Ltd, Welwyn Garden City, UK; ${ }^{4}$ Royal BromptonHospital, London, UK

\subsection{6/thoraxjnl-2014-206260.173}

Objectives Idiopathic pulmonary fibrosis (IPF) is associated with aberrant expression of signalling pathways involved in embryonic development, including the Hedgehog ( $\mathrm{Hh}$ ) pathway. $\mathrm{Hh}$ can promote multiple profibrotic processes including myofibroblast differentiation, expression of extracellular matrix genes, migration, and survival. Vismodegib is a small-molecule inhibitor of the Hedgehog (Hh) signalling pathway approved for the treatment of basal cell carcinoma. We sought to evaluate the activity of Hh signalling in IPF lung tissue and identify blood biomarkers of Hh pathway activity in IPF patients.

Methods Gene expression in biopsies from IPF and control unused donor lungs, and in fibroblasts stimulated with Shh in vitro. CXCL14 protein was measured in plasma from IPF patients and from solid tumour patients treated with vismodegib in a phase $1 \mathrm{~b}$ clinical trial (NCT00968981).

Results We observed significantly increased expression levels of Hh pathway genes including SMO, PTCH2, GLI1, and GLI2 in IPF vs control lungs. To identify candidate systemic biomarkers of Hh pathway activity, we compared transcriptional data from IPF lung biopsies and fibroblasts stimulated in vitro with Shh. The gene most significantly upregulated in both datasets was CXCL14, which encodes a soluble secreted chemokine whose expression is inhibited in vitro by the addition of vismodegib. Circulating CXCL14 protein levels were significantly higher in plasma from IPF patients than controls. In solid tumour patients, circulating CXCL14 levels were significantly reduced upon treatment with vismodegib.

Conclusions We observed strong evidence for Hedgehog pathway activity in IPF lungs. CXCL14 is a systemic biomarker that could be used to identify IPF patients with increased Hh pathway activity and monitor the pharmacodynamic effects of vismodegib in IPF. The ISLAND trial is a phase 2 randomised, doubleblind, placebo-controlled study designed to evaluate the safety and efficacy of vismodegib in IPF patients. ISLAND will enrol 129 patients with IPF, randomised 2:1 to vismodegib or placebo for 60 weeks. The primary efficacy objective is to evaluate the effect of vismodegib on mean change in forced vital capacity (FVC). Secondary outcome measures include IPF and/or Hhassociated biomarkers, progression-free survival, and change in quantitative lung fibrosis on HRCT. Enrollment is expected to start in October 2014. 